\title{
Determinação da relação entre vazões líquida e sólida e análise de fatores que influenciam a dinâmica do transporte de sedimentos na Bacia Hidrográfica do Rio Jordão (UPGRH-PN 1)
}

Determination of the liquid and solid discharge ratio and analysis of factors influencing the dynamics of sediment transport in the Jordão River Watershed (UPGRH-PN 1)

\section{Rodrigo de Almeida Oliveira Peixoto ${ }^{\top}$, Carlos Eugenio Pereira'* ${ }^{1}$, Marcio Ricardo Salla ${ }^{1}$, José Eduardo Alamy Filho' $\bullet$, Abner Nishimura' $₫$}

\begin{abstract}
RESUMO
O entendimento do escoamento de sedimentos é fundamental para a adequada gestão e uso dos recursos hídricos. A falta de planejamento de uso e ocupação do solo, marcado por ações antrópicas irracionais, influencia diretamente a dinâmica dos sedimentos nas bacias hidrográficas em todo o território nacional. Nesse sentido, o presente artigo teve como objetivos determinar as descargas de sedimentos, analisar fatores que podem influenciar o carreamento de sedimentos na bacia hidrográfica e construir as curvas-chave que representam a relação entre as descargas sólida e líquida no rio Jordão. O estudo foi realizado na Bacia Hidrográfica do Rio Jordão, estabelecendo-se quatro estações de amostragem, sendo realizadas nove campanhas de medições de vazões e coletas de água para análise de sedimentos suspensos em épocas secas e chuvosas no período de agosto de 2017 a julho de 2018. As concentrações e descargas de sedimentos apresentaram variações entre as estações e os períodos de amostragem. $\mathrm{Na}$ época chuvosa, os valores das descargas e concentrações foram superiores ao período de estiagem. A maior concentração média foi de 101,16 mg...-1 na estação 3, e a maior descarga sólida total ocorreu na estação 4, com valor médio de 113,83 t.d'. Além da precipitação, fatores como cobertura vegetal, uso do solo e declividade influenciam o carreamento dos sedimentos no rio Jordão. As curvaschave de sedimentos em suspensão e totais, para cada estação e para os dados unidos de todas as estações, apresentaram boas correlações.
\end{abstract}

Palavras-chave: sedimentologia; método de Colby; descarga de sedimentos; Bacia Hidrográfica do Rio Jordão.

\begin{abstract}
Understanding the sediment flow is fundamental for the proper management and use of the water resources. The lack of planning in the use and occupation of land, marked by irrational anthropogenic actions, directly influences the sediment dynamics in river basins throughout the national territory. In this sense, the objectives of this article were to determine sediment discharges, to analyze factors that can influence sediment dynamics in the basin and to construct the key curves that represent the relationship between the solid and liquid flow of the Jordão River. The study was carried out in the Jordão River Basin. Four sampling stations were established, and nine campaigns were carried out to measure flows and water samples for the analysis of suspended sediments during dry and rainy seasons from August 2017 to July 2018. The concentrations and discharges of sediment presented variations between the seasons and sampling periods. In the rainy season, the values of discharges and concentrations were higher than in the dry season. The highest mean concentration was 101.16 mg.L'-1at station 3, and the highest total solid discharge occurred at station 4 with a mean value of 113.83 t.d' ${ }^{-1}$. In addition to precipitation, factors such as vegetation cover, land use and slope are influencing the sediment dynamics in the Jordão River. The key curves of suspended and total sediments obtained for each season and the bound data from all stations showed good correlations.
\end{abstract}

Keywords: sedimentology; Colby’s method; discharge of sediments; Jordão River Watershed.

\section{INTRODUÇÃO}

A erosão, o transporte e a deposição de sedimentos são fenômenos naturais do meio ambiente e que têm sido intensificados e acelerados significativamente

pela atividade humana (BRITO et al., 2009). As ações irracionais do homem afetam o equilíbrio da natureza. O desmatamento, a poluição e a degradação excessiva da mata, do solo e da água impactam o ambiente de forma negativa.

Universidade Federal de Uberlândia - Uberlândia (MG), Brasil.

*Autor correspondente: cepereira@ufu.br

Recebido: 19/09/2018 - Aceito: 11/09/2019 - Reg. ABES: 20180137 
Entre os principais fatores relacionados com o aumento da degradação ambiental, estão as frequentes alterações não planejadas do uso da terra, acima da capacidade de suporte do solo. A falta de planejamento da bacia hidrográfica e o uso inadequado de técnicas influenciam diretamente a dinâmica dos sedimentos na bacia hidrográfica, favorecendo a erosão dos solos e o aumento da carga de sedimentos no rio (CARVALHO; BRUMATTI; DIAS, 2012).

Após ocorrer a erosão, esses sedimentos chegam ao corpo hídrico, onde são transportados em suspensão ou pelo leito do canal. Segundo Carvalho (2008), a produção e o transporte de sedimentos ocorrem principalmente em época chuvosa ou durante fortes precipitações, correspondendo a cerca de $80 \%$ de todo sedimento transportado no curso de água. Além de fatores como a precipitação, Mohammad et al. (2016) e Silva et al. (2005) expõem que a carga de sedimentos nos rios é influenciada pelo tipo de solo, pela cobertura vegetal, pelo uso da terra e pela topografia da bacia hidrográfica.

A análise eficiente e o entendimento do transporte de sedimentos, junto à descarga líquida de cursos hídricos, são essenciais. As descargas líquida e sólida afetam aspectos ambientais, econômicos e sociais inerentes à população que depende dos recursos naturais da bacia. Para Cobaner, Unal e Kisi (2009), o estudo do transporte de sedimentos em rios é muito importante, pois fatores como poluição da água, assoreamento de rios, diminuição da vida útil de reservatórios, comprometimento de equipamentos, construções de hidrelétricas, navegabilidade, entre outros, são alguns problemas influenciados por esse processo.

O conhecimento do comportamento e da quantidade dos sedimentos nos corpos hídricos de uma bacia hidrográfica é fundamental para a adequada gestão e uso de seus recursos hídricos, além de ser essencial para o desenvolvimento de projetos hidráulicos. Acompanhar o escoamento de sedimentos permite ainda o diagnóstico de eventuais impactos em sua área de drenagem ao longo do tempo, podendo se tornar um importante indicador ambiental (CRISPIM et al., 2015).

O entendimento das redes de rios naturais atrai a atenção das comunidades científicas. $\mathrm{O}$ transporte de sedimentos em um rio natural é um processo transitório em razão das mudanças no escoamento, a qualquer momento, causado por eventos aleatórios (BERGHOUT; MEDDI, 2016). Vários modelos são desenvolvidos para estimar a quantidade de sedimentos transportados, conhecida como descarga sólida, com o objetivo de obter compreensão mais profunda da dinâmica do transporte de sedimentos e dos eventos que influenciam esse transporte (WANG et al., 2008). Apesar dos diversos modelos de estimativa, ainda não existe um método universal em razão da complexidade e das inúmeras variáveis que influenciam o processo.

No Brasil, as pesquisas relacionadas ao assunto são essenciais, pois a energia elétrica fundamental para o desenvolvimento do país é gerada principalmente por usinas hidrelétricas, que apresentam reservatórios sujeitos a assoreamento e turbinas propensas à abrasão por partículas suspensas. De acordo com a Agência Nacional de Energia Elétrica (ANEEL, 2018), mais de $60 \%$ da produção anual de eletricidade do Brasil é proveniente de fontes hidráulicas.

A Bacia Hidrográfica do Rio Jordão, área de estudo desta pesquisa, está inserida no contexto apresentado por apresentar nove eixos para execução de Pequenas Centrais Hidrelétricas (PCHs), dos quais quatro estão aceitos e cinco têm o eixo disponível, segundo informações da ANEEL (2018). Além disso, a região apresenta evidências da ocorrência de processo erosivo e transporte de sedimentos.

Considerando a grande importância do assunto, as escassas informações sobre o aporte e a descarga de sedimentos na Bacia Hidrográfica do Rio Jordão, e que o conhecimento do comportamento hidrossedimentológico é fundamental para a adequada gestão e uso dos recursos hídricos, este trabalho teve como objetivos determinar as descargas sólidas suspensas e totais pelo método de Colby (1957), analisar os fatores que influenciam o carreamento de sedimentos na bacia hidrográfica e construir as curvas-chave que representam a relação entre as descargas líquida e sólida no rio Jordão.

\section{METODOLOGIA}

\section{Área de estudo}

O presente trabalho foi realizado na Bacia Hidrográfica do Rio Jordão, localizada na mesorregião do Triângulo Mineiro e Alto Paranaíba, na Unidade de Planejamento e Gestão dos Recursos Hídricos-Alto Rio Paranaíba 1 (UPGRH-PN1), entre as coordenadas geográficas $18^{\circ} 25^{\prime} 41^{\prime \prime}$ e $18^{\circ} 50^{\prime} 51^{\prime \prime}$ de latitude Sul e $47^{\circ} 49^{\prime} 47^{\prime \prime}$ e $48^{\circ} 12^{\prime} 51^{\prime \prime}$ de longitude Oeste (SALLA et al., 2013). A área da bacia tem aproximadamente $926 \mathrm{~km}^{2}$, com extensão do curso de água principal de aproximadamente $90 \mathrm{~km}$ da nascente até a confluência com o rio Paranaíba.

As estações de monitoramento foram definidas a partir do planejamento e estudo do curso do rio. Quatro estações de amostragem foram estabelecidas, localizadas nas seguintes coordenadas geográficas:

- Estação 1: 1841'33,02”S e 4805'39,7”W;

- Estação 2: 18³6'57,19”S e 4805'46,03”'W;

- Estação 3: 18³3'37,94”'S e 4806'49,58”'W;

- Estação 4: 18²6'28,83”S e 4805’43,99”W.

A bacia hidrográfica, junto ao trecho do rio e às estações de amostragem, estão representados na Figura 1.

Neste estudo, foram realizadas nove campanhas de medições de vazão e coletas de água para análise de sedimentos suspensos, nos períodos de seca e chuva, entre os meses de agosto de 2017 e julho de 2018.

\section{Determinação da vazão líquida}

Para a determinação da seção transversal, da velocidade do escoamento e da vazão líquida do curso de água foram utilizados os equipamentos 
ADCP (Acoustic Doppler Current Profiler) ou molinete fluviométrico. A utilização de dois equipamentos é explicada pela diferença de profundidade das seções nos períodos distintos de coleta de dados. O ADCP foi utilizado nas seções e épocas que o nível líquido possibilitava a travessia transversal do equipamento, pois este tem a limitação de nível de água mínimo de $0,30 \mathrm{~m}$. Assim, era necessário utilizar o molinete para a obtenção de dados.
Nos trabalhos realizados em campo, o ADCP foi fixado em uma corda para facilitar sua travessia entre as margens do rio nas estações fluviométricas, e por meio do programa fornecido pelo fabricante, River Surveyor Stationary Live, instalado em um computador portátil, cuja conexão é via bluetooth ao equipamento, foi possível registrar os resultados de vazão em tempo real. Na sequência, foram realizadas no mínimo três travessias em cada seção amostrada do rio. A seção



Figura 1 - Mapa representativo da localização da Bacia Hidrográfica do Rio Jordão e do trecho de amostragem. 
transversal do leito do rio, os perfis e as direções de velocidades foram registrados e o cálculo da vazão em tempo real realizado pelo programa.

O molinete fluviométrico utilizado apresenta uma hélice dinâmica que gira sob ação do movimento da água do rio. Em campo, foi utilizada uma corda graduada para a realização das leituras de metro em metro com as verticais fixadas, formando subáreas verticais ao longo da seção transversal real do curso de água. Para a leitura, em cada metro, o molinete foi posicionado seguindo as recomendações de acordo com a profundidade do rio. Para obtenção da velocidade do escoamento, o molinete foi conectado a um contador de giros controlado por um cronômetro digital que relaciona o número de rotação por segundo com a velocidade do escoamento, calculada por meio da Equação 1.

\section{$\mathrm{V}=\mathrm{A} \cdot \mathrm{N}+\mathrm{B}$}

Em que:

$\mathrm{V}=$ velocidade da água $(\mathrm{m} / \mathrm{s})$;

$\mathrm{N}$ = número de rotação por segundo;

$\mathrm{A}$ e $\mathrm{B}=$ constantes que individualizam cada aparelho aferido.

A vazão (qi) foi determinada pelo produto da área molhada da seção escolhida pela velocidade média (v) do escoamento da corrente líquida. $\mathrm{O}$ cálculo da área molhada foi obtido pela medida da profundidade em várias verticais escalonadas. Cada elemento de área $(\mathrm{Ai})$ foi calculado como retângulo ou trapézio de acordo com a forma da seção topo batimétrica. Calculou-se a qi em cada Ai utilizando-se a Equação 2:

$q \mathrm{i}=\mathrm{V} \cdot \mathrm{Ai}$

A vazão total $(\mathrm{Q})$ foi obtida pela somatória das qi em cada $\mathrm{Ai}$, conforme a Equação 3:

$\mathrm{Q}=\sum \mathrm{qi}$

\section{Amostragem e análise de sedimentos suspensos}

A coleta de sedimentos suspensos foi realizada pelo método indireto de amostragem por igual incremento de largura - IIL, com o auxílio do amostrador integrador de série Norte-Americana (U.S-DH 48), com bocal de coleta na frente do amostrador de diâmetro igual a 1/8". Durante a amostragem, a temperatura da água e a do ar foram medidas utilizando termômetros. A seção transversal do rio foi dividida em segmentos de larguras iguais com a seleção de 10 a 20 verticais, e as coletas foram realizadas com a mesma velocidade de trânsito na vertical. O volume de amostra coletada foi de $2 \mathrm{~L}$.

No Laboratório de Saneamento da Faculdade de Engenharia Civil da Universidade Federal de Uberlândia (LABSAN-FECIV-UFU) foram realizadas as análises da concentração de sedimentos em suspensão pelo método de filtração, descrito por Carvalho (2008). Em cada cadinho foi colocada uma membrana de fibra de vidro de $0,45 \mu \mathrm{m}$, com a parte rugosa para baixo. Para aderência da membrana no cadinho, foi necessário filtrar uma pequena quantidade de água destilada. Após a filtração, o cadinho com a fibra foi colocado na estufa $\left(105 \pm 2^{\circ} \mathrm{C}\right)$ por 15 minutos e, em seguida, levado ao forno mufla $\left(550 \pm 50^{\circ} \mathrm{C}\right)$ por 30 minutos. A partir desse instante, o cadinho era resfriado até temperatura ambiente e pesado (P1). As amostras foram homogeneizadas em um béquer e filtradas no cadinho, por meio do sistema de filtração a vácuo com a utilização de bomba de vácuo e Kitassato. O procedimento de filtração foi realizado com toda a amostra coletada. Em seguida, o cadinho contendo os sólidos suspensos retidos no filtro foi levado para estufa $\left(105 \pm 2^{\circ} \mathrm{C}\right)$. Ao atingir peso constante, o cadinho foi resfriado em um dessecador até a temperatura ambiente para a pesagem (P2).

A determinação da concentração de sedimentos suspensos (Css) foi realizada pela Equação 4 .

$\mathrm{Css}=\frac{\mathrm{P} 2-\mathrm{P} 1}{\mathrm{~V}} .1000$

Em que:

Css $=$ concentração de sedimentos suspensos $\left(\mathrm{mg} \cdot \mathrm{L}^{-1}\right)$;

$\mathrm{P} 1$ = tara do cadinho $(\mathrm{g})$;

P2 = pesagem do cadinho com amostra após secagem (g);

$\mathrm{V}=$ volume da amostra $(\mathrm{L})$

Com a realização das análises de sedimentos suspensos e as medições de vazão, calculou-se a descarga de sedimentos suspensos (Qss) de cada seção analisada, a partir da Equação 5.

Qss $=0,0864 \cdot$ Q.Css

Em que:

Qss = descarga de sedimentos suspensos $\left(\mathrm{t}^{\mathrm{d}} \mathrm{d}^{-1}\right)$;

$\mathrm{Q}=$ vazão total da seção $\left(\mathrm{m}^{3} \cdot \mathrm{s}^{-1}\right)$;

Css $=$ concentração de sedimentos suspensos $\left(\mathrm{mg} \cdot \mathrm{L}^{-1}\right)$.

Para estimar a descarga de sedimentos total (Qst) foi utilizado o método de Colby (1957), que se baseia no método modificado de Einstein e em várias medições de campo, seguindo a metodologia descrita por Carvalho (2008). O método basicamente utiliza três ábacos e os dados de profundidade média, largura da seção, velocidade média, descarga líquida e Css. Segundo Costa (2012), o método é bastante aplicado em estudos desenvolvidos no Brasil.

A Qst foi calculada a partir da Equação 6.

Qst $=$ Qss + Qnm 
Em que:

Qst = descarga de sedimentos total $\left(\mathrm{t} . \mathrm{d}^{-1}\right)$;

Qnm $=$ descarga de sedimento não medida $\left(\mathrm{t} \cdot \mathrm{d}^{-1}\right)$.

A descarga de sedimento não medida (Qnm) corresponde à descarga de arrasto somada à descarga não amostrada devido a limitações do equipamento de amostragem. A Qnm foi determinada com o auxílio dos ábacos e pela Equação 7 .

\section{Qnm = qnm.K.L}

Em que:

qnm = descarga sólida não medida aproximada por metro de largura $\left(\mathrm{t} . \mathrm{d}^{-1} \cdot \mathrm{m}^{-1}\right)$;

$\mathrm{K}$ = fator de correção (adimensional);

$\mathrm{L}=\operatorname{largura}(\mathrm{m})$.

Após a determinação dos valores da Qss e da Qst pelo método de Colby, foram traçadas as curvas-chave de sedimentos para cada estação, utilizando a metodologia proposta por Carvalho et al. (2000). As curvas-chave de sedimentos são representadas geralmente em forma de potência, como indicado na Equação 8.

(Qst ou Qss)=a. Q
Em que:

a e $\mathrm{n}=$ parâmetros ajustados para cada estação;

$\mathrm{Q}=\operatorname{vazão}$ total $\left(\mathrm{m}^{3} \cdot \mathrm{s}^{-1}\right)$.

A identificação do uso, da ocupação e da cobertura da terra na Bacia Hidrográfica do Rio Jordão foi feita com a utilização dos programas Quantum GIS - QGIS 2.18.4 e Multispec. O mapeamento foi realizado com base em imagens atuais do satélite LANDSAT-8, obtidas diretamente no site do Instituto Nacional de Pesquisas Espaciais (INPE, 2018). No QGIS, as imagens das diferentes bandas foram georreferenciadas a partir do sistema de referência SIRGAS 2000, sendo recortadas utilizando o shape da bacia hidrográfica como camada máscara. No Multispec, foi realizada a junção de todas as imagens recortadas das diferentes bandas para realizar a classificação supervisionada.

\section{RESULTADOS E DISCUSSÃO}

Com as nove campanhas de medições realizadas no rio Jordão, entre os meses de agosto de 2017 e maio de 2018, foram obtidos dados de largura, profundidade, velocidade e vazão para cada estação, como apresentados na Tabela 1. Na região de estudo, os meses de agosto a outubro e o mês de maio correspondem a períodos de estiagem; já os meses de novembro a março compõem o período chuvoso. No dia 22 de maio de 2018, data da última campanha, não foi possível a coleta

Tabela 1 - Dados de medições realizadas nas estações 1, 2, 3 e 4.

\begin{tabular}{|c|c|c|c|c|c|c|c|c|c|c|c|}
\hline \multicolumn{2}{|l|}{ Período } & \multicolumn{3}{|c|}{ Seco } & \multicolumn{5}{|c|}{ Chuvoso } & \multirow{2}{*}{$\frac{\text { Seco }}{\text { Maio } 18}$} & \multirow{2}{*}{ Média } \\
\hline Estações & $\begin{array}{c}\text { Mês/Ano } \\
\text { Dados }\end{array}$ & Ago. 17 & Set. 17 & Out. 17 & Nov. 17 & Dez. 17 & Jan. 18 & Fev. 18 & Mar. 18 & & \\
\hline 1 & \multirow{4}{*}{$L(m)$} & 14,0 & 14,0 & 14,0 & 15,4 & 15,9 & 15,0 & 15,1 & 15,5 & 15,0 & 14,9 \\
\hline 2 & & 14,0 & 15,5 & 15,0 & 15,5 & 16,0 & 16,3 & 16,4 & 16,0 & - & 15,6 \\
\hline 3 & & 15,0 & 15,0 & 15,0 & 16,8 & 17,0 & 16,5 & 16,0 & 16,5 & 15,3 & 15,9 \\
\hline 4 & & 15,0 & 15,0 & 16,0 & 16,3 & 16,0 & 16,3 & 17,0 & 17,0 & 17,0 & 16,2 \\
\hline 1 & \multirow{4}{*}{$P(m)$} & 0,411 & 0,401 & 0,401 & 0,454 & 0,698 & 0,439 & 0,531 & 0,467 & 0,477 & 0,5 \\
\hline 2 & & 0,500 & 0,430 & 0,458 & 0,568 & 0,772 & 0,605 & 0,615 & 0,608 & - & 0,6 \\
\hline 3 & & 0,909 & 0,858 & 0,869 & 0,705 & 0,946 & 0,891 & 0,901 & 0,941 & 0,865 & 0,9 \\
\hline 4 & & 0,317 & 0,230 & 0,235 & 0,573 & 1,018 & 0,821 & 0,859 & 0,700 & 0,672 & 0,6 \\
\hline 1 & \multirow{4}{*}{$V\left(m . s^{-1}\right)$} & 0,256 & 0,200 & 0,201 & 0,340 & 0,518 & 0,361 & 0,409 & 0,358 & 0,279 & 0,3 \\
\hline 2 & & 0,500 & 0,430 & 0,458 & 0,568 & 0,772 & 0,605 & 0,615 & 0,608 & - & 0,6 \\
\hline 3 & & 0,350 & 0,319 & 0,323 & 0,387 & 0,685 & 0,460 & 0,395 & 0,460 & 0,372 & 0,4 \\
\hline 4 & & 0,437 & 0,412 & 0,389 & 0,499 & 0,795 & 0,508 & 0,561 & 0,490 & 0,454 & 0,5 \\
\hline 1 & \multirow{4}{*}{$\mathrm{Q}\left(\mathrm{m}^{3} \cdot \mathrm{s}^{-1}\right)$} & 0,725 & 0,450 & 0,491 & 3,289 & 6,118 & 2,637 & 4,300 & 2,159 & 1,850 & 2,4 \\
\hline 2 & & 1,461 & 1,053 & 0,600 & 5,204 & 11,927 & 6,046 & 7,560 & 4,814 & - & 4,8 \\
\hline 3 & & 2,100 & 1,400 & 1,450 & 5,858 & 14,223 & 7,913 & 8,663 & 6,918 & 4,778 & 5,9 \\
\hline 4 & & 2,164 & 1,506 & 1,535 & 6,921 & 16,111 & 8,655 & 10,905 & 6,948 & 5,174 & 6,7 \\
\hline
\end{tabular}

L: largura; P: profundidade média; V: velocidade média da água; Q: vazão, 
de amostras na estação 2, devido a dificuldades de acesso ao local. Os resultados das estações estão apresentados na Tabela 1.

A variação dos dados apresentados é explicada pelos diferentes períodos de amostragem. Os valores de vazão no rio aumentaram significativamente no período chuvoso. Enquanto, no período seco, as vazões determinadas nas diferentes estações ficaram na faixa de $0,45 \mathrm{a} 2,16 \mathrm{~m}^{3} \cdot \mathrm{s}^{-1}$, nos meses chuvosos, as vazões foram de 2,16 a $16,11 \mathrm{~m}^{3} \cdot \mathrm{s}^{-1}$. Além da vazão, os dados de largura, profundidade e velocidade foram utilizados para o cálculo da descarga de sedimento pelo método de Colby (1957).

Os resultados de Css, Qss, Qnm e Qst são apresentados nas Tabelas 2 e 3.
Analisando as descargas de sedimentos, a estação 1 apresentou menores valores de Qss e Qst, com médias de 17,31 e 30,57 t.d ${ }^{-1}$, respectivamente. Os maiores valores ocorreram na estação 4 , com média de Qss igual a 64,64 t.d $\mathrm{d}^{-1}$ e de Qst de 113,83 t.d $\mathrm{d}^{-1}$. Em todas as campanhas os valores máximos foram obtidos na estação 3, com Qss de 165,31 t.d ${ }^{-1}$ e Qst de 418,87 t.d ${ }^{-1}$. Quando analisadas todas as estações em conjunto, a média de todos os valores de Qss foi de 46,83 t.d ${ }^{-1} \mathrm{e}$ de Qst, 80,51 t.d ${ }^{-1}$.

A estação 3 apresentou os maiores valores de Css, com máximo de 206,33 mg.L $\mathrm{L}^{-1}$ e mínimo de 10,68 mg.L $\mathrm{L}^{-1}$, sendo valor médio e desvio

Tabela 2 - Resultados de concentração e descarga líquida e de sedimentos no rio Jordão.

\begin{tabular}{|c|c|c|c|c|c|c|c|c|c|c|}
\hline \multirow{2}{*}{ Período } & \multicolumn{5}{|c|}{ Estação 1} & \multicolumn{5}{|c|}{ Estação 2} \\
\hline & $\operatorname{Css}\left(m g . L^{-1}\right)$ & $\mathrm{Q}\left(\mathrm{m}^{3} \cdot \mathrm{s}^{-1}\right)$ & Qss (t.d $\left.{ }^{-1}\right)$ & Qnm (t.d. $\left.{ }^{-1}\right)$ & Qst (t.d $\left.{ }^{-1}\right)$ & Css (mg. $\left.\mathrm{L}^{-1}\right)$ & $\mathrm{Q}\left(\mathrm{m}^{3} \cdot \mathrm{s}^{-1}\right)$ & Qss (t.d $\left.d^{-1}\right)$ & Qnm (t.d $\left.{ }^{-1}\right)$ & Qst (t.d $\left.{ }^{-1}\right)$ \\
\hline \multirow{3}{*}{ Seco } & 10,67 & 0,725 & 0,668 & 1,937 & 2,605 & 6,89 & 1,461 & 0,870 & 0,686 & 1,556 \\
\hline & 32,44 & 0,450 & 1,261 & 1,750 & 3,012 & 37,78 & 1,053 & 3,437 & 0,776 & 4,213 \\
\hline & 18,67 & 0,491 & 0,792 & 1,354 & 2,146 & 23,11 & 0,600 & 1,198 & 0,844 & 2,042 \\
\hline \multirow{5}{*}{ Chuvoso } & 87,33 & 3,289 & 24,817 & 13,278 & 38,096 & 102,67 & 5,204 & 46,162 & 27,124 & 73,286 \\
\hline & 113,00 & 6,118 & 59,731 & 48,981 & 108,712 & 92,22 & 11,927 & 95,034 & 124,091 & 219,125 \\
\hline & 105,56 & 2,637 & 24,049 & 14,858 & 38,907 & 73,33 & 6,046 & 38,307 & 27,366 & 65,674 \\
\hline & 73,00 & 4,300 & 27,121 & 19,021 & 46,142 & 87,26 & 7,560 & 56,995 & 52,311 & 109,305 \\
\hline & 63,11 & 2,159 & 11,772 & 13,046 & 24,818 & 102,67 & 4,814 & 42,703 & 34,959 & 77,663 \\
\hline Seco & 35,00 & 1,850 & 5,594 & 5,082 & 10,677 & - & - & - & - & - \\
\hline Média & 59,86 & 2,45 & 17,31 & 13,26 & 30,57 & 65,74 & 4,83 & 35,59 & 33,52 & 69,11 \\
\hline Máximo & 113,00 & 6,12 & 59,73 & 48,98 & 108,71 & 102,67 & 11,93 & 95,03 & 124,09 & 219,13 \\
\hline Mínimo & 10,67 & 0,45 & 0,67 & 1,35 & 2,15 & 6,89 & 0,60 & 0,87 & 0,69 & 1,56 \\
\hline Desvio padrão & 37,64 & 1,90 & 19,28 & 14,92 & 34,01 & 37,82 & 3,83 & 32,91 & 41,15 & 73,19 \\
\hline
\end{tabular}

Css: concentração de sedimentos suspensos; Q: vazão; Qss: descarga de sedimentos suspensos; Qnm: descarga de sedimento não medida; Qst: descarga de sedimentos total.

Tabela 3 - Resultados de concentração e descarga líquida e de sedimentos no rio Jordão.

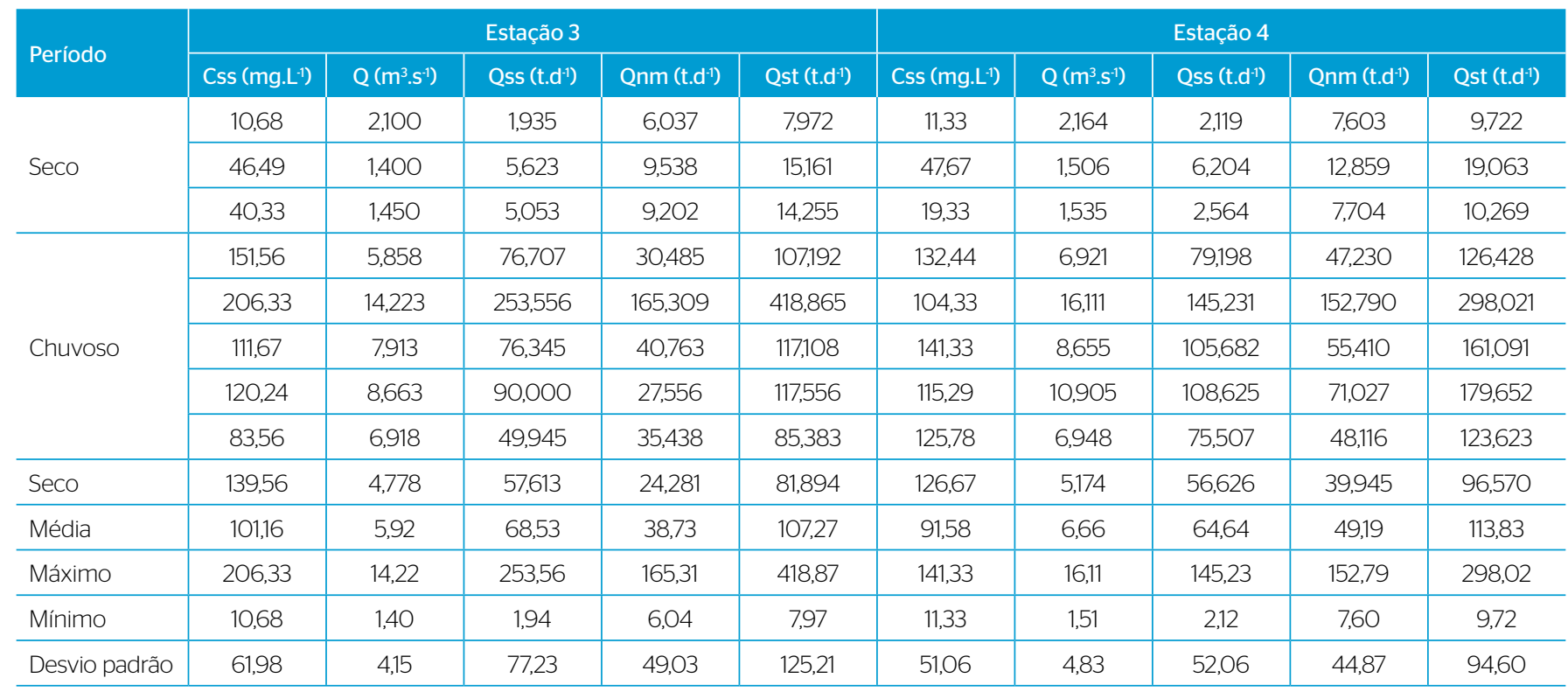

Css: concentração de sedimentos suspensos; Q: vazão; Qss: descarga de sedimentos suspensos; Qnm: descarga de sedimento não medida; Qst: descarga de sedimentos total. 
padrão de 101,16 e 61,98 mg. $\mathrm{L}^{-1}$, respectivamente, para vazão média de $5,92 \mathrm{~m}^{3} \cdot \mathrm{s}^{-1}$. Já a estação 4 apresentou maiores valores de vazões com média de $6,66 \mathrm{~m}^{3} \cdot \mathrm{s}^{-1}$, o que é explicado devido aos diversos afluentes que incrementam a vazão ao longo do percurso do rio. Nessa seção, a Css média foi de 91,58 mg.L-1 , com valores na faixa de 11,33 a 141,33 mg.L.'-

Os valores de Css obtidos nas estações de amostragem no rio Jordão diferem dos valores encontrados por outros autores nos diferentes cursos de água estudados. Silva Junior et al. (2011) obtiveram no Riacho Mimoso, em Pernambuco, concentração média de 212,43 mg. $\mathrm{L}^{-1}$ para vazão entre $0,049 \mathrm{e} 11,59 \mathrm{~m}^{3} \cdot \mathrm{s}^{-1}$. Lima et al. (2005) apresentaram em seu estudo valores de Css na faixa de 197,58 a 386,25 mg.L $\mathrm{L}^{-1}$ e vazão média de $609 \mathrm{~m}^{3} \cdot \mathrm{s}^{-1}$ em quatro estações do rio Doce. Grove et al. (2015), no estudo realizado em diversos cursos de água no Reino Unido, obtiveram diferentes concentrações e vazões médias. No rio Bodilly, a concentração foi de $29,65 \mathrm{mg} \cdot \mathrm{L}^{-1}$ para vazão de $0,22 \mathrm{~m}^{3} \cdot \mathrm{s}^{-1}$, enquanto no rio Stincharo, o valor de Css foi de 14,6 mg. $\mathrm{L}^{-1}$ e a vazão, 2,99 $\mathrm{m}^{3} \cdot \mathrm{s}^{-1}$. Garrido et al. (2018), no rio Piranhas, na Paraíba, encontraram concentração de 46,59 mg.L $\mathrm{L}^{-1}$ para vazão média de $12,87 \mathrm{~m}^{3} \cdot \mathrm{s}^{-1}$.

Analisando todas as seções em conjunto, fica evidente que a concentração de sedimento aumenta no período chuvoso, conforme apresentado na Figura 2. O valor médio das quatro estações é de aproximadamente $100 \mathrm{mg} \cdot \mathrm{L}^{-1}$ na época chuvosa, enquanto no período de estiagem a média não passou de $60 \mathrm{mg} \cdot \mathrm{L}^{-1}$. O aumento da concentração em períodos de maiores precipitações também foi identificado por Silva Junior et al. (2011), Grove et al. (2015), Mohammad et al. (2016) e Garrido et al. (2018).

A variação dos valores de concentração de sedimentos em cada estação está sendo influenciada pelos diferentes uso e ocupação do solo nos locais mais próximos a cada seção. Com os programas Quantum GIS (QGIS) e Multispec, foi possível analisar os diferentes usos do solo da bacia hidrográfica, como expresso na Tabela 4.

A estação 3, que exibiu maior média de concentração e maiores valores de concentrações em cinco campanhas, como evidenciado na Figura 2, não apresenta Área de Preservação Permanente (APP),

Tabela 4 - Uso e ocupação do solo na Bacia Hidrográfica do Rio Jordão.

\begin{tabular}{l|c|c}
\hline Uso e ocupação & Área (ha) & Porcentagem (\%) \\
\hline Vegetação & $22.259,31$ & 24,01 \\
\hline Agricultura & $38.285,70$ & 41,30 \\
\hline Pastagem & $26.222,92$ & 28,29 \\
\hline Área urbana & $2.669,22$ & 2,88 \\
\hline Solo exposto & $3.253,84$ & 3,51 \\
\hline Total & 92.691 & 100 \\
\hline
\end{tabular}

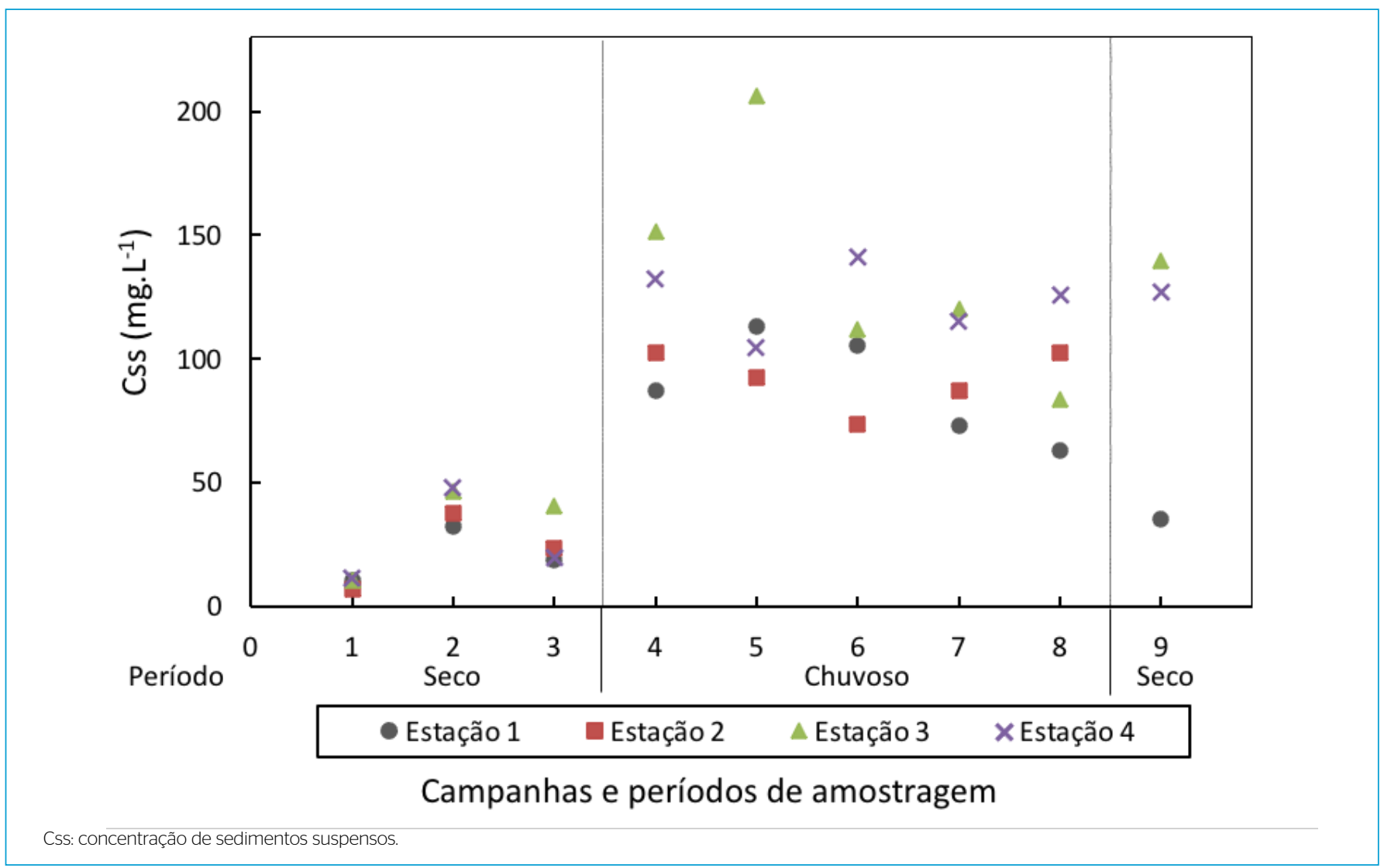

Figura 2 - Concentração de sedimentos em suspensão nas estações nos diferentes períodos de amostragem. 
pois sua vegetação foi substituída por áreas de agricultura e pastagem. As demais estações apresentam uma pequena quantidade de cobertura vegetal nas margens, porém não é suficiente para conter a degradação do solo e, principalmente, a chegada dos sedimentos em épocas chuvosas. Carvalho (2008), Vanzela, Hernandez e Franco (2010), Cabral e Reis (2015) e Aparecido et al. (2016) salientaram a grande influência do uso do solo na concentração e na carga de sedimentos no corpo hídrico.

A Bacia Hidrográfica do Rio Jordão é predominantemente ocupada por agricultura (mais de $41 \%$ de sua área). A pastagem vem em segundo lugar, com $28,29 \%$, seguida da vegetação, com aproximadamente $24 \%$. A área urbana e o solo exposto representam as regiões com menor porcentagem de área.

Além de afetar as diferentes concentrações em cada estação, a substituição da vegetação por áreas agrícolas e de pastagem está influenciando no aumento da carga de sedimentos, principalmente em períodos chuvosos. Com a retirada da vegetação nas épocas de precipitação, ocorre maior escoamento superficial, menor infiltração e, consequentemente, maior perda de solo e aumento da carga de sedimentos no rio. Zhang et al. (2011) obtiveram resultados similares, mostrando que a maior intensidade de precipitação e a menor cobertura vegetal resultaram em maiores cargas de sedimentos no corpo hídrico.

A retirada sem planejamento da vegetação deixa o solo desprotegido e aumenta os processos erosivos pela intensificação do escoamento superficial. Arcova e Cicco (1997) já salientavam que a degradação da vegetação e o uso agrícola das bacias hidrográficas contribuem para a intensificação do processo de assoreamento e erosão das margens dos cursos hídricos, além de comprometer o equilíbrio do ecossistema, a qualidade da água e aumentar o transporte de sedimentos nos rios.

O perfil longitudinal do trecho de amostragem, apresentado na Figura 3, é outro fator que pode influenciar a variação espacial da carga de sedimentos no rio Jordão. Por meio do perfil, verifica-se que as estações 1 e 2 se situam na parte mais acentuada, com maior declividade. Já as regiões de médio e baixo curso, representadas pelas estações $3 \mathrm{e}$ 4, apresentam menores declividades. Em razão da maior declividade no alto curso do rio, as partículas são transportadas para regiões mais baixas, promovendo o aumento da carga de sedimentos transportada nas estações 3 e 4 .

Com base nos resultados de Qss e Qst, apresentados nas Tabelas 2 e 3, foi possível obter as curvas-chave de sedimentos, mostrando a correlação entre as descargas sólida e líquida do rio Jordão. A Figura 4 representa as curvas-chave de sedimentos suspensos e total, a equação e o coeficiente de determinação $\left(\mathrm{R}^{2}\right)$ de cada estação considerada neste estudo.

Todas as estações de amostragem apresentaram boas correlações, com valores de $\mathrm{R}^{2}$ maiores que 0,87 . A correlação entre a Qss e a $\mathrm{Q}$ obteve $\mathrm{R}^{2}$ menores quando comparados aos valores de $\mathrm{R}^{2}$ obtidos da correlação entre a Qst e a Q. Morais (2015), Silva Junior et al. (2011) e Garrido et al. (2018) também apresentaram bons valores de $\mathrm{R}^{2}$.

Os dados de todas as estações foram unidos para avaliar a distribuição geral dos resultados de Qss e Qst, em função da vazão. Assim, obteve-se uma distribuição bastante semelhante quando analisada cada estação separadamente. Por fim, as curvas-chave da Qss e da Qst do rio Jordão foram ajustadas, conforme apresentado nas Figuras 5 e 6.

O ajuste da curva-chave de sedimentos em suspensão apresentou boa correlação, com $\mathrm{R}^{2}$ de 0,8974 e parâmetros de ajuste iguais a 2,1934 e 1,7053 . Outros autores alcançaram melhores $\mathrm{R}^{2}$, como é o caso de Poletto et al. (2011), que obtiveram $\mathrm{R}^{2}$ de 0,9882 no rio São Francisco Falso, no estado do Paraná.

O ajuste da curva-chave de sedimentos total apresentou boa correlação, com $\mathrm{R}^{2}$ de 0,9173 , um pouco melhor do que a curva de sedimentos suspensos. Os parâmetros de ajuste foram iguais a 5,3395 e 1,5396.

\section{CONCLUSÕES}

As concentrações e descargas de sedimentos obtidas entre agosto de 2017 e maio de 2018, no rio Jordão, apresentaram variações entre as

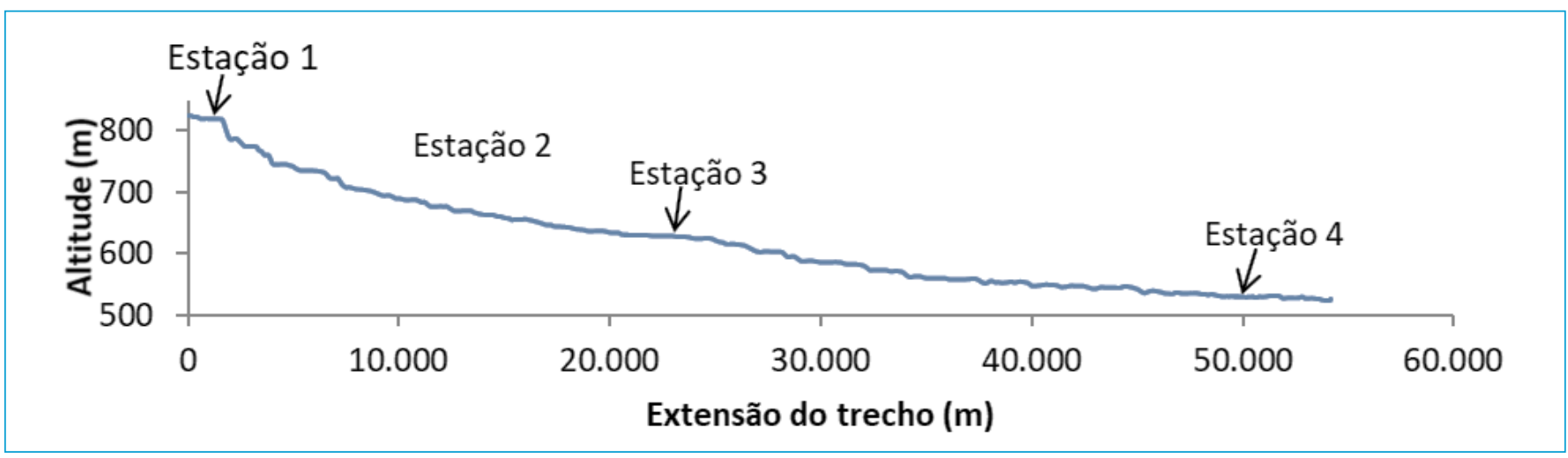

Figura 3 - Perfil longitudinal do trecho de amostragem do rio Jordão. 
estações e os períodos de amostragem. Na época chuvosa, os valores obtidos de concentrações foram maiores do que no período de estiagem, evidenciando a influência da precipitação na quantidade de sedimentos no rio Jordão. Entre as estações, a maior concentração média foi de 101,16 mg.L - $^{-1}$ na estação 3 e a maior Qst ocorreu na estação 4, com valor médio de 113,83 t.d $\mathrm{d}^{-1}$.

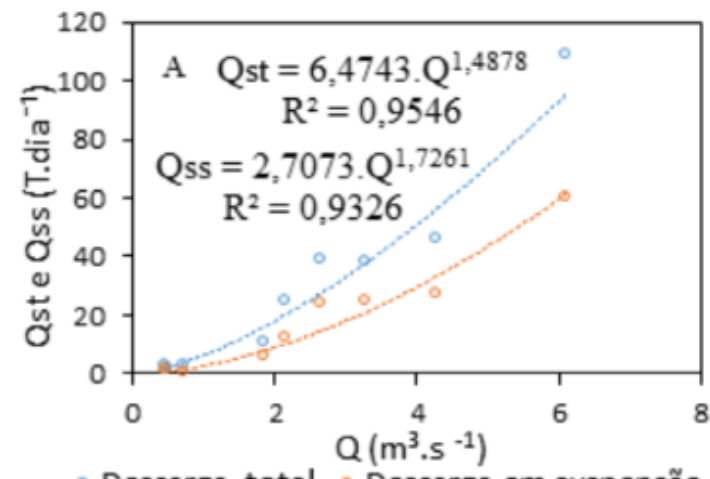

- Descarga total - Descarga em suspensão

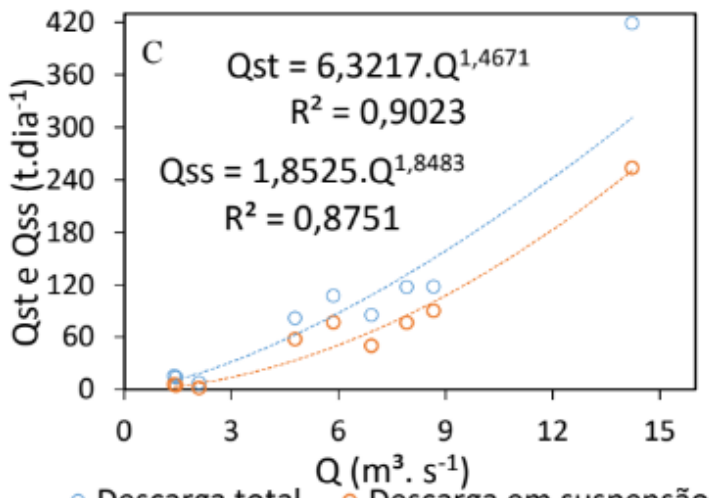

- Descarga total $\circ$ Descarga em suspensão
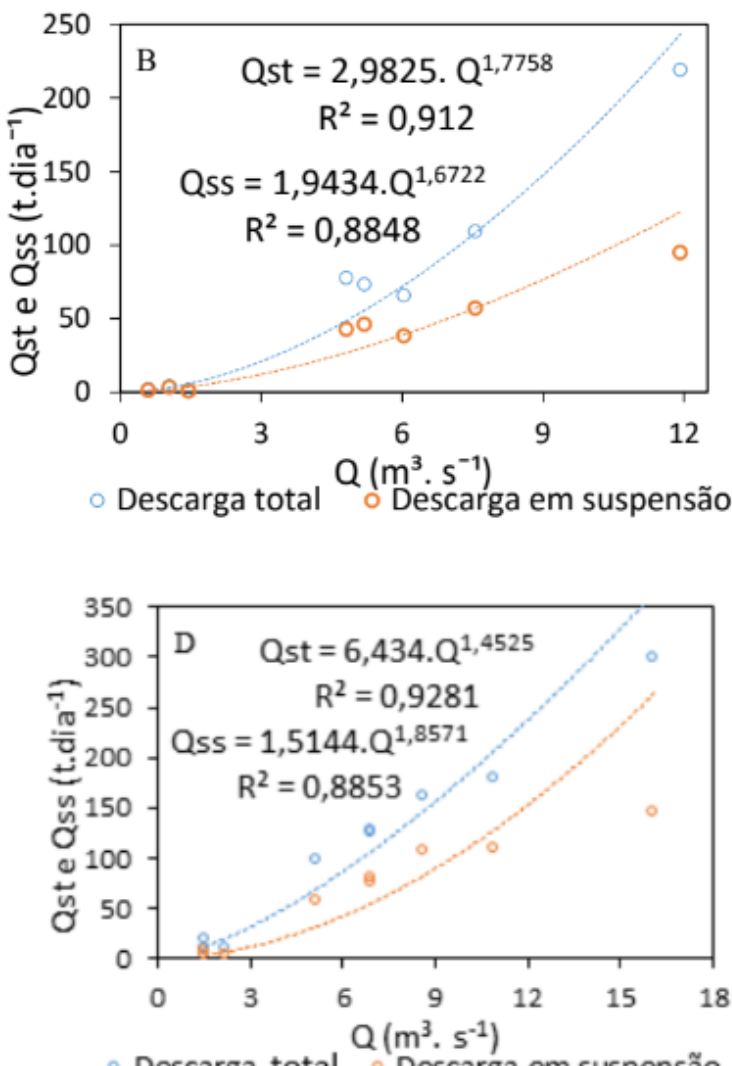

Descarga total Descarga em suspensão

Qst: descarga de sedimentos total; Qss: descarga de sedimentos suspensos; R²: coeficiente de determinação; Q: vazão total.

Figura 4 - Curva-chave de sedimentos nas estações do rio Jordão. (A) Estação 1; (B) Estação 2; (C) Estação 3; e (D) Estação 4.
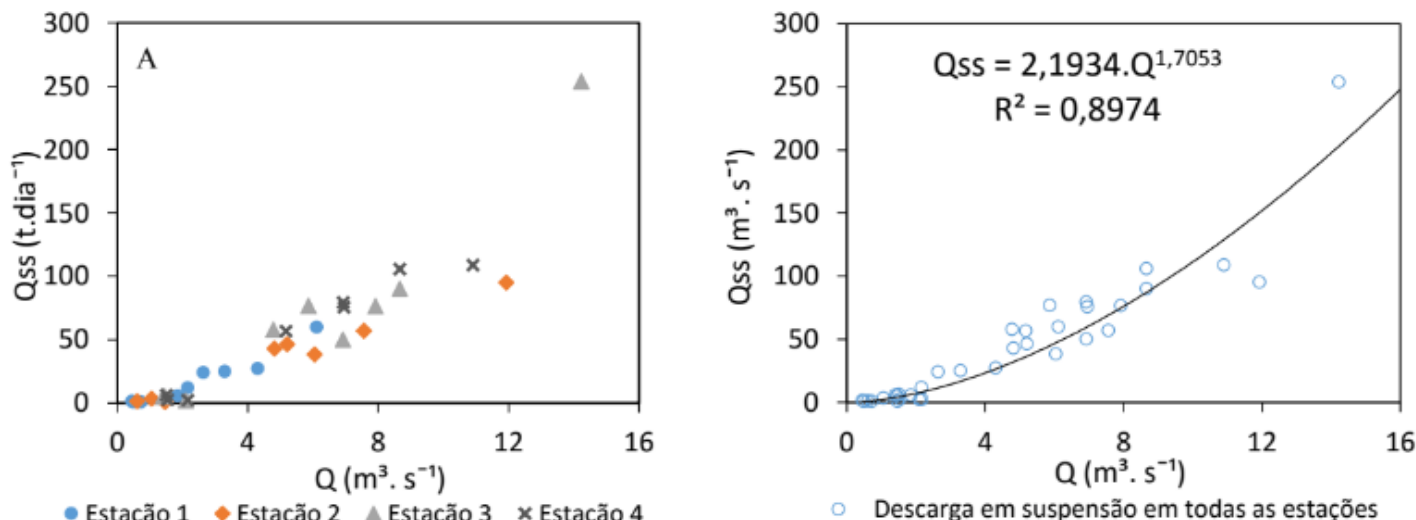

Qss: descarga de sedimentos suspensos; R²: coeficiente de determinação; Q: vazão total.

Figura 5 - Relação entre a descarga sólida em suspensão e a vazão do rio Jordão. (A) Distribuição de dados por estação; (B) curva-chave de sedimentos. 

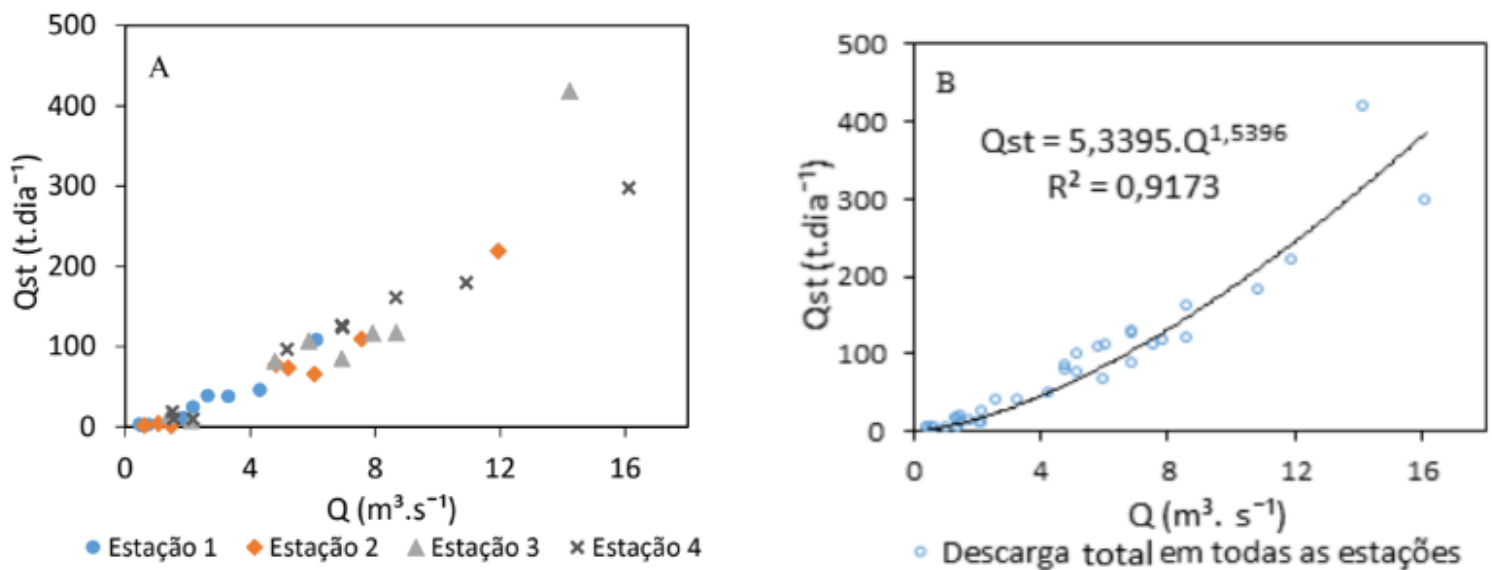

Qst: descarga de sedimentos total; R²: coeficiente de determinação; Q: vazão total.

Figura 6 - Relação entre descarga sólida total e vazão do rio Jordão. (A) Distribuição de dados por estação; (B) curva-chave de sedimentos.

Além da precipitação, fatores como cobertura vegetal, uso do solo e declividade estão influenciando a dinâmica dos sedimentos no rio Jordão. A retirada da cobertura vegetal afeta diretamente na quantidade e no transporte de partículas no rio. Em razão da grande quantidade de áreas agrícolas e de pastagem na bacia hidrográfica, o rio Jordão, em épocas de maiores precipitações, apresenta elevadas concentrações e carga de sedimentos. A declividade acentuada nas regiões de alto curso está promovendo o caminhamento de sedimentos para as estações 3 e 4, que apresentam maiores cargas de sedimentos.

As curvas-chave de sedimentos em suspensão e totais obtidas para cada estação e para os dados unidos de todas as estações apresentaram bons $\mathrm{R}^{2}$, sendo 0,8751 o menor valor, para a curva de descarga em suspensão da estação 3, e 0,9543 o maior valor, para a curva de descarga total da estação 1. O bom ajuste dos parâmetros e a obtenção de valores de $R^{2}$ próximos a 1 são essenciais para viabilizar a utilização das curvas-chave de sedimentos.
A falta de planejamento de uso e ocupação do solo está influenciando diretamente a dinâmica do sedimento na bacia hidrográfica, favorecendo a erosão dos solos e o aumento da carga de sedimentos no rio. Nesse contexto, o estudo foi importante por fornecer subsídios para tomada de decisão dos setores competentes, além de contribuir para o melhor entendimento da concentração e das descargas de sedimentos no rio Jordão.

\section{AGRADECIMENTOS}

À Fundação de Amparo à Pesquisa do Estado de Minas Gerais (FAPEMIG) o apoio financeiro ao projeto de pesquisa APQ 3899/2016, à Coordenação de Aperfeiçoamento de Pessoal de Nível Superior (CAPES) a bolsa de mestrado e à Faculdade de Engenharia Civil (FECIV) da Universidade Federal de Uberlândia (UFU) o transporte durante as coletas de amostras e dados em campo.

\section{REFERÊNCIAS}

AGÊNCIA NACIONAL DE ENERGIA ELÉTRICA (ANEEL). Carteira. ANEEL. Disponível em: <http://www.aneel.gov.br/scg/Doc/Carteira. xlsx>. Acesso em: 25 jul. 2018.

APARECIDO, C.F.F.; VANZELA, L.S.; VAZQUEZ, G.H.; LIMA, R.C. (2016) Manejo de bacias hidrográficas e sua influência sobre os recursos hídricos. Brazilian Journal of Irrigation and Drainage, Botucatu, v. 21, n. 2, p. 239-256. https://doi.org/10.15809/irriga.2016v21n2p239-256

ARCOVA, F.C.S.; CICCO, V. (1997) Características do deflúvio de duas microbacias hidrográficas no laboratório de hidrologia florestal Walter Emmench, Cunha - SP. Revista do Instituto Florestal de São Paulo, São Paulo, v. 9, n. 2, p. 153-170.
BERGHOUT, A.; MEDDI, M. (2016) Sediment transport modelling in wadi Chemora during flood flow events. Journal of Water and Land Development, n. 31, p. 23-31. https://doi.org/10.1515/jwid-2016-0033

BRITO, R.N.R.; ASP, N.E.; BEASLEY, C.R.; SANTOS, H.S.S. (2009) Características Sedimentares Fluviais Associadas ao Grau de Preservação da Mata Ciliar - Rio Urumajó, Nordeste Paraense. Acta Amazônica, v. 39, n. 1, p. 173-180. https://doi.org/10.1590/S004459672009000100017

CABRAL, S.L.; REIS, R.S. (2015) Influência do uso e ocupação do solo na produção de sedimentos na bacia do rio jacarecica. Revista de Geografia, Pernambuco, v. 32, n. 2, p. 147-157. 
CARVALHO, A.P.V.; BRUMATTI, D.V.; DIAS, H.C.T. (2O12) Importância do manejo da bacia hidrográfica e da determinação de processos hidrológicos. Revista Brasileira de Agropecuária Sustentável, v. 2, n. 2. p. 148-156. https://doi.org/10.21206/rbas.v2i2.177

CARVALHO, N.O. (2008) Hidrossedimentologia Prática. 2a ed. Rio de Janeiro: Interciência. 599 p.

CARVALHO, N.O.; FILIZOLA JÚNIOR, N.P.; SANTOS, P.M.C.; LIMA, J.E.F.W. (2000) Guia de práticas sedimentométricas. Brasília: ANEEL. 154 p. Disponível em: <https://www2.aneel.gov.br/biblioteca/ downloads/livros/Guia_prat_port.pdf>. Acesso em: 17 jun. 2018.

COBANER, M.; UNAL, B.; KISI, O. (2009) Suspended sediment concentration estimation by na adaptive neuro-fuzzyand neural network approaches using hydro-meteorological data. Journal of Hydrology, v. 367, n. 1-2, p. 52-61. https://doi.org/10.1016/j. jhydrol.2008.12.024

COLBY, B.R. (1957) Relationship of unmeasured sediment discharge to mean velocity. Transactions American Geophysical Union, v. 38, n. 5, p. 708-717. https://doi.org/10.1029/TR038i005p00708

COSTA, L.F.(2012) Análise de sensibilidade dos parâmetros utilizados na determinação de taxas de assoreamento de reservatórios. $124 \mathrm{f}$. Dissertação (Mestrado em Engenharia Civil) - Programa de PósGraduação em Engenharia Civil, Universidade Federal do Rio de Janeiro, Rio de Janeiro.

CRISPIM, D.L.; ISMAEL, L.L.; SOUZA, T.M.I.; GARRIDO, JW.A.; QUEIROZ, M.M.F. (2015) Transporte e caracterização de sedimentos de fundo no rio piranhas em uma seção de controle próximo à sede do município de pombal-PB. Holos, v. 3, p. 93-101. https://doi. org/10.15628/holos.2015.2518

GARRIDO, J.W.A.; SOUSA, T.M.l.; ISMAEL, L.L.; CRISPIM, D.L.; FERREIRA, P.M.L.; QUEIROZ, M.M.F. (2018) Determinação das curvas-chave de descargas de sedimentos em suspensão na bacia hidrográfica do Rio Piancó-Piranhas-Açu. Engenharia Sanitaria e Ambiental, v. 23, n. 1, p. 69-75. https://doi.org/10.1590/ s1413-41522018138711

GROVE, M.K.; BILOTTA, G.S.; WOOCKMAN, R.R.; SCHWARTZ, J.S. (2015) Suspended sediment regimes in contrasting referencecondition freshwater ecosystems: Implications for water quality guidelines and management. Science of the Total Environment, v. 502, p. 481-492. https://doi.org/10.1016/.j.scitotenv.2014.09.054

INSTITUTO NACIONAL DE PESQUISAS ESPACIAIS (INPE). Catálogo. INPE. Disponível em: <http://www.dgi.inpe.br/catalogo/>. Acesso em: 2 fev. 2018
LIMA, J.E.F.W:; LOPES, W.T.A.; CARVALHO, N.O; VIEIRA, M.R.; SILVA, E.M. (2005) Suspended sediment fluxes in the large river basins of Brazil. In: SEDIMENT BUDGETS, 1., 2005, Foz do Iguaçu. Publication of International Association of Hydrological Sciences... v. 291, p. 355-363.

MOHAMMAD, M.E.; AL-ANSARI, N.; ISSA, I.E.; KNUTSSON, S. (2O16) Sediment in Mosul Dam reservoir using the HEC-RAS model. Lakes \& Reservoirs: Research \& Management, v. 21, n. 3, p. 235-244. https:// doi.org/10.1111//re.12142

MORAIS, R.C.S. (2015) Estimativa de produção e transporte de sedimentos na bacia hidrográfica do Rio Parnaíba, nordeste do Brasil. Revista Equador, Piauí, v. 4, n. 4, p. 81-97.

POLETTO, K.; SAMPAIO, S.C:; QUEIROZ, M.M.F.; GOMES, B.M.; SONCELA, R. (2011) Turbidimetry as an alternative method to determine the rating curve of suspended sediments. Engenharia Agrícola, Jaboticabal, v. 31 n. 3, p. 598-606. https://doi.org/10.1590/S0100-69162011000300019

SALLA, M.R.; PEREIRA, C.E.; ALAMY FILHO, J.E.; PAULA, L.M.; PINHEIRO, A.M. (2013) Estudo da autodepuração do Rio Jordão, localizado na bacia hidrográfica do Rio Dourados. Engenharia Sanitária e Ambiental, Rio de Janeiro, v. 18, n. 2, p. 105-114. https://doi. org/10.1590/S1413-41522013000200002

SILVA, D.D; PRUSKI, F.F; SCHAEFER, C.E.G.R; AMORIM, R.S.S.; PAIVA K.W.N. (2015) Efeito da cobertura nas perdas de solo em um Argissolo Vermelho-Amareloutilizandosimulador dechuva. EngenhariaAgrícola, v. 25, n. 2, p. 409-419. https://doi.org/10.1590/SO100-69162005000200014

SILVA JUNIOR, V.P.; MONTENEGRO, A.A.A.; SILVA, T.P.N.; GUERRA S.M.S.; SANTOS, E.S. (2O11) Produção deágua e sedimentos em bacia representativa do semiárido pernambucano. Revista Brasileira de Engenharia Agrícola e Ambiental, Campina Grande, v. 15, n. 10, p. 1073-1081. https://doi.org/10.1590/S1415-43662011001000012

VANZELA, L.S.;HERNANDEZ,F.B.T.;FRANCO, R.A.M.(2010) Influência do uso e ocupação do solo nos recursos hídricos do Córrego Três Barras, Marinópolis. Revista Brasileira de Engenharia Agrícola e Ambiental, v. 14, n. 1, p. 55-64. https://doi.org/10.1590/S1415-43662010000100008

WANG, X.; HAO, R.; HUO, J.; ZHANG, J. (2008) Modeling sediment transport in river networks. Physica A: Statistical Mechanics and its Applications, v. 387, n. 25, p. 6421-6430. https://doi.org/10.1016/j. physa.2008.07.027

ZHANG, G.H.; LIU, G.B.; WANG, G.L.; WANG, Y.X. (2011) Effects of Vegetation Cover and Rainfall Intensity on Sediment-Bound Nutrient Loss, Size Composition and Volume Fractal Dimension of Sediment Particles. Pedosphere, v. 21, n. 5, p. 676-684. https://doi. org/10.1016/S1002-0160(11)60170-7 